\title{
The Effectiveness of Using Breathing Exercise on Sleep Quality Among Hospitalized Patients
}

\author{
Eman Fadl Abd El Khalik', Marwa Mohammad Abd Elbaky ${ }^{2,}$, , Naglaa Ahmed Ahmed ${ }^{3}$, \\ Sahar Hamza Taha Moursy ${ }^{1}$ \\ ${ }^{1}$ Department of Medical Surgical Nursing, Faculty of Nursing, Minia University, Minia City, Egypt \\ ${ }^{2}$ Department of Medical Surgical Nursing (Critical Care Nursing), Faculty of Nursing, Minia University, Minia City, Egypt \\ ${ }^{3}$ Department of Critical Care and Emergency Nursing, Faculty of Nursing, Assuit University, Assuit City, Egypt
}

\section{Email address:}

marwa.radwan1@mu.edu.eg (M. M. A. Elbaky)

${ }^{*}$ Corresponding author

\section{To cite this article:}

Eman Fadl Abd El Khalik, Marwa Mohammad Abd Elbaky, Naglaa Ahmed Ahmed, Sahar Hamza Taha Moursy. The Effectiveness of Using Breathing Exercise on Sleep Quality Among Hospitalized Patients. American Journal of Nursing Science. Special Issue: Evidence Based Nursing. Vol. 9, No. 4, 2020, pp. 267-275. doi: 10.11648/j.ajns.20200904.28

Received: May 19, 2020; Accepted: July 3, 2020; Published: July 22, 2020

\begin{abstract}
Sleep disturbance among hospitalized patients has many factors especially at critical care units. Daily breathing exercises as pursed lip breathing (PLB) and diaphragmatic breathing improves sleep quality and reduces the occurrence of delirium. Aim of the study Evaluate the effectiveness of using breathing exercise on sleep quality among hospitalized patients. Design quasi experimental design. Sample A purposive sample including 152 patients were divided into four equal groups two from the Medical ward (study and control) 38 patients each group and two form the Medical Intensive Care Unit (MICU) (study and control) 38 each group at Minia university hospital. Results majority of the groups were male; all patients were in the middle age. (39\% and $44.7 \%$ ) of the medical ward groups (study and control) and (52.6\% and $44.7 \%$ ) respectively of the MICU groups had sever sleep problem. But after applying breathing exercise for 3 continuous days, the study groups (44.7\%) of the medical ward and (50\%) of the MICU had great sleep quality. Majority of study group's patients at the medical ward and MICU had no Confusion," with percentage (60.5\% and 52.6\%) respectively which mean no presence of delirium. Conclusion breathing exercise had significance effect on improving sleeping quality and reduce the occurrence of delirium among the study groups than the control groups.
\end{abstract}

Keywords: Effectiveness, Breathing Exercise, Sleep, Quality and Hospitalized Patients

\section{Introduction}

Sleep is a vital process for human, it is influenced by many biological and environmental factors. Sleep affects health, welfare and quality of life because it can promote healing, immune function and recovery from diseases. Sleep disturbance is one of the most serious complains that disturb many hospitalized patients especially patients in Intensive Care Units (ICU) [1]. Sleep stages, including non-rapid eye movement (NREM) and rapid eye movement (REM) [2]. Research has shown that, prevalence of sleep disturbance among critical ill and hospitalized patients has been shown to be more than $(50 \%)$, and of the remaining sleep time only $3.0 \%$ to $4.0 \%$ in REM stage [3]. In another study High prevalence of poor sleep quality in hospitalized patients to be ranging between $43 \%$ and $91 \%$ [4].

Hospitalized patients, and those whom are critical, were known to have severe disturbed sleep quality. Seep quality in critical ill patient is characterized by a predominance of wakefulness and light sleep (sleep stages I and II), and a relative lack of rapid eye movement (REM) and deep sleep (delta sleep, formerly referred to as non-REM sleep stages III/IV). Sleep deprivation is known to lead to several clinical and physiologic manifestations and side effects [5].

Despite the importance of sleep, the assessment of sleep quality does not form part of standard clinical nursing practice in many ICUs or general wards. Continuous sleep quality assessment among hospitalized patients has been 
strongly recommended in order to discover the most side effects of inadequate sleep [6]. Sleep deprivation among hospitalized patients may contribute to adverse in-hospital and post-discharge side effects, such as delirium, respiratory dysfunction, stimulating cardiovascular attack. It increases blood pressure, heart rate, immune system dysfunction, impaired resistance to infection, alterations in nitrogen balance and impaired wound healing. Also increase in activities of sympathetic system, due to lack of sleep, causes increase in the pain experience and there is a direct relationship between quality of sleep and pain $[6,7]$.

Many factors contribute to sleep deprivation and disturbance among critical ill patients, this factors are divided to patient related factors as type and severity of the underlying illness, pathophysiology of the acute illness, abnormal physiological symptoms as dyspnea, hypoxia, fever, neurological dysfunction, anxiety/fear, depression and others. [8].

Environmental factors as noise in ICU which raised from multiple sources, including alarms, mechanical ventilators, pagers, televisions, and monitoring equipment. Therapeutic interventions as unit medications. Procedures related factors as vital signs, blood draws, diagnostic tests, and hygienic intervention. Staff related factors such as nursing procedures, beepers, phone, overhead paging and staff conversation [9].

Some studies suspect that there will be a connection between poor sleep quality and the occurrence of delirium. Delirium occurs among hospitalized patients especially those at ICUs. Delirium is considered an acute reversible disorder, it is a disturbance of the consciousness with inattention accompanied by a change in cognition or perceptual disturbance that develops over a short period (hours to days) and fluctuates over time [10].

A possible link between both sleep deprivation and delirium has been hypothesized in terms of a common pathophysiologic pathway, shared mechanisms, and a potential relationship. On the other hand, a few literatures suggested that occurrence of sleep deprivation could lead to delirium [11].

Breathing exercises are a complementary and nonpharmacological therapy to reduce many patient's related problems as sleep deprivation, cardiac autonomic function, depression, anxiety, high blood pressure, and lung diseases. It has been shown that breathing exercise stimulate body and brain function, and sympathetic-parasympathetic systems function, considered an excellent tool to facilitate relaxation [12].

Daily breathing exercises can improve sleep quality. Breathing exercises for hospitalized patients can strength the breathing muscles, increase the entrance of more oxygen during breath with less effort and promotes relaxation. The value from breathing exercises as pursed lip breathing (PLB) and diaphragmatic breathing are improving exercise tolerance and helps in better sleep quality and quality of life [13].

\subsection{Significant of the Study}

Disturbances of sleep quality is very common problem among hospitalized patients. It is widely documented that patients in the intensive care unit (ICU) suffer from poor sleep with up to $61 \%$ of patients reporting sleeping problems. [poor sleep could have major consequences for overall wellbeing. Even though the consequences on clinical outcome remain partly unknown, strong correlations between ICU sleep disruption and increased incidence of systemic illness, poor recovery, delayed wound healing and increased mortality [14].

Sleep deprivation leads to occurrence of inattention, fluctuating mental status, disorganized thinking, mood changes and an altered level of consciousness, this finding which are also characteristics of patients with delirium. So that it is likely suspected that reducing sleep disturbance using breathing exercise could have a better effect on the patient outcome and will reduce delirium. The problem is the misdiagnosed patient to detect presence of sleep deprivation and alleviate the factors which lead to its occurrence [15].

So that researchers conduct this study to assess sleep quality among hospitalized patients and improving it using breathing exercise which suspected to reduce delirium in order to improve patient's health status and reduce hospital stay and mortality.

\subsection{The Aim of the Study}

Evaluate the effectiveness of using breathing exercise on sleep quality among hospitalized patients.

\section{Methods}

\subsection{Quasi Experimental Research Design Used to Fulfill the Aim of This Study Subjects}

A purposive sample including 152 patients was divided into four equal groups two groups of patients were taken from the Medical general ward (study and control) 38 each group. Another two groups form the Medical Intensive Care Unit (MICU) (study and control) 38 patients in each group. the study was conducted at the Minia University Hospital, in Minia city, Egypt. The sample was collected at cross section period of six months from the first of April 2019 to the end of September 2019.

The study sample included the following patients were conscious adult, from both genders, Medical patients, who spent more than two nights in the hospital (ward or ICU). The study Excluded the following: Patients connected with invasive mechanical ventilation, comatose, under sedation regimen, mental handicapped, and refused to participate.

This paper will hypothesize that: using breathing exercise will improve sleep quality among hospitalized patients and improving sleep quality will reduce delirium and improve patient outcome.

\subsection{Data Collection Tools}

Three tools will be designed and used for collecting data for this study, these tools were prepared and tested by researcher after extensive literature review Tool one: Patient Health Assessment Questioner: This tool developed and 
filled by the researcher during patient interview, it consisted two parts, Part I: Socio- demographic data as age, sex, marital status and level of education, smoking and occupation. Part II: patient medical data as medical diagnosis, past medical history and presence of chronic disease.

Tool two: includes two parts: Part I: Sleep Quality Questionnaire (SQQ) used to assess the quality of sleep pattern among hospitalized patients developed by Colin Espie and adopted from Ibáñez, V., Silva, J., \& Cauli, O. [16] that consisted of (9) multiple choice questions, each question of the SQQ consisted from a five choices each choice take score from (0 to 4). The total score of the SQQ was calculated and defined as: if the patient takes score from $(0$ 9) the patient had sleep problems seem to be severe. patient should definitely try to get some help, (10-18) patient had some sleep problems. It's important to examine his sleeping habits and see how nurse can make changes, (19-27) the patient's sleep is in good shape, but there were still many steps he can take to make it even better and (28-36) the patient's sleep is in great shape. Part II: Factors affecting sleep quality assessment sheet developed by the researcher to assess the factors that lead to sleep deprivation among hospitalized patients it included three domains (patient related factors, staff, and environmental factors).

Tool three: Neelon and Champagne Confusion Scale (NEECHAM) is scale, originally designed to detect confusion and delirium in hospitalized patients, adopted from Neelon, V. J., et al [17]. This scale consisted of nine-item scale is separated into three different categories: (a) ability to process information (e.g., attention, command, orientation), (b) behavior (e.g., appearance, motor and verbal), and (c) physiological condition (e.g., vital signs, oxygen saturation, and episodes of incontinence). The overall score of the NEECHAM ranges from 0 through 30 points. A score of 30 indicates that the patient gives a maximal (normal) reaction and 0 indicates a minimal reaction. The scale gives four grades of outcome: moderate to severe confusion and/or delirium (0-19 points), mild to early confusion and/or delirium (20-24 points), 'not confused' but at high risk of confusion and/or delirium (25-26 points), and normal cognitive functioning i.e. absence of confusion and/or delirium (27-30 points).

\subsection{Validity and Reliability}

Study tools were tested for content validity by a jury panel of (5) experts in nursing and medicine. A pilot study was done on $10 \%$ (15) of patients to test the feasibility of the study and applicability of the tool and there are no modifications done.

\subsection{Ethical Consideration}

An official permission from the research ethics committee of the faculty of nursing, Minia University, Egypt was obtained to conduct the study. Official permissions were granted from the director of Minia Chest and Cardiothoracic hospital and the head of the Medical department and MICU. Also official permissions were granted from the head nurses of the medical ward and MICU to conduct the study. Written informed consent was obtained by a researcher from the patients after an explanation of the aim of the study. Confidentiality of data, privacy, voluntary participation and right to refuse to participate in the study were informed to them by the researchers through personal communication.

\subsection{Research Procedure}

The researchers assessed all critical ill patients from all groups whom spent more than 24 hours at the medical ward and medical intensive care unit after they became clinical stable, and before applying the breathing exercise, the researchers assessed their sociodemographic data, medical diagnosis, and past medical history using tool one (part I and II). The researchers conducted an interview with the patients to assess their sleep quality using Sleep Quality Questionnaire (SQQ) (part I in tool two). Then the researchers assessed the factors that led to sleep deprivation using the part II of the same tool.

Both study groups (at the MICU and the medical general ward) were taught breathing exercise by the researchers after spending 24 hours of admission to the MICU or medical ward. Each patient from the study groups received explanation about pursed lib breathing and diaphragmatic breathing.

In pursed lib breathing (PLB) the researchers instructed patients from the study groups inhale through the nose while counting of " 1 to 2 ", the researchers instructed the patients to observe the stomach muscles to move outward as air goes into the lungs. Exhale through pursed lips (as if you are whistling or blowing out a candle). Each patient from the study groups were advised to repeat the PLB ten times (ten PLB) in an hour with a few seconds pause in between.

Diaphragmatic breathing is intended to help patient use the diaphragm correctly while breathing to strengthen the diaphragm, decrease the work of breathing by slowing breathing rate decrease oxygen demand, use less effort and energy to breathe. Patients were advised to Sit comfortably, with the knees bent and the shoulders, relaxed. Breathe in slowly through the nose so that the stomach moves out against the hand. The hand on the chest should remain as still as possible. Place one hand on the upper chest and the other just below the rib cage. This will allow patient to feel the diaphragm move as he or she breathe. Tighten the stomach muscles, letting them fall inward as patient exhale through pursed lips. Also each patient from the study groups were advised to repeat the diaphragmatic breathing ten times in an hour with a few seconds pause in between.

Both the PLB and the diaphragmatic breathing sessions were repeated every 3 hours during the day. Each time the patient done the breathing exercise was documented in his patient's record to be able to follow up him. The patients of the study group were instructed to do the breathing exercise for three continuous days while they were in the MICU and medical ward. Finally, the patients of the intervention groups and control groups were evaluated again to assess their sleep 
quality using tool two ( $1^{\text {st }}$ part $)$ and the effect of sleep quality on the presence of confusion and delirium using tool three (Neelon and Champagne Confusion Scale NEECHAM) at the fourth day of the study.

\subsection{Statistical Analysis}

Statistical package for the social sciences (SPSS version 20; SPSS Inc., Chicago, Illinois, USA) software was used to evaluate the data obtained from the study. The statistics were presented as mean $\pm \mathrm{SD}$ and frequency tables. Correlations between the quality of sleep and other variables were determined using Pearson's correlation coefficients analysis. $P$ value of less than 0.05 was accepted as statistically significant.

\section{Result}

Table 1 Demonstrates the majority of the groups were male at the medical ward and the MICU except the control group at the MICU (52\%) of the patients were female. All patients were in the middle age group with mean and standard deviation (S. D) $(37.3 \pm 12.5 \& 45.8 \pm 9.5)$ at the medical ward and $(37.8 \pm 13.638 .3 \pm 13.2)$ at the MICU (study and control) respectively. The highest percentage of patients at the medical ward were chronic disease cases (55.3 $\& 71.1)$ respectively unlike patients at the MICU more than half of the patients were acute $(55.2 \& 52)$ respectively.

Table 1. Distribution of the Socio demographic characteristic for both study \& groups subjects among Medical Word and MICU patients. (N=152).

\begin{tabular}{|c|c|c|c|c|}
\hline \multirow{3}{*}{ Demographic data } & \multicolumn{4}{|c|}{ Medical ward MICU } \\
\hline & \multicolumn{2}{|c|}{ Study Control } & \multicolumn{2}{|c|}{ Study Control } \\
\hline & No $(\%)$ & No $(\%)$ & No $(\%)$ & No $(\%)$ \\
\hline \multicolumn{5}{|l|}{ Age / years } \\
\hline$<20$ years & $3(7.9)$ & $0(0)$ & $6(15.8)$ & $3(7.9)$ \\
\hline $20-40$ years & $22(57.9)$ & $21(55.3)$ & $16(42.1)$ & $20(52)$ \\
\hline$>40$ years & $13(34.2)$ & $17(44.7)$ & $16(42.1)$ & $15(39.4)$ \\
\hline Mean \pm SD years & \multicolumn{2}{|c|}{$37.3 \pm 12.545 .8 \pm 9.5$} & \multicolumn{2}{|c|}{$37.8 \pm 13.638 .3 \pm 13.2$} \\
\hline \multicolumn{5}{|l|}{ Gender } \\
\hline Male & $24(63.2)$ & $27(71.1)$ & $28(73.6)$ & $18(47.3)$ \\
\hline Female & $14(36.8)$ & $11(28.9)$ & $10(26.3)$ & $20(52.6)$ \\
\hline \multicolumn{5}{|l|}{ Education level } \\
\hline Illiterate & $10(26)$ & $13(34.2)$ & $8(21)$ & $10(26)$ \\
\hline Read and write & $7(1.8)$ & $10(26)$ & $13(34.2)$ & $7(1.8)$ \\
\hline Secondary school & $17(44.7)$ & $3(7.8)$ & $10(26)$ & $9(23.6)$ \\
\hline University & $4(10.5)$ & $12(31.5)$ & $7(1.8)$ & $12(31.5)$ \\
\hline \multicolumn{5}{|l|}{ Medical Diagnosis } \\
\hline Acute & $17(44.7)$ & $11(28.9)$ & $21(55.2)$ & $20(52)$ \\
\hline Chronic & $21(55.3)$ & $27(71.1)$ & $17(44.7)$ & $18(47.3)$ \\
\hline
\end{tabular}

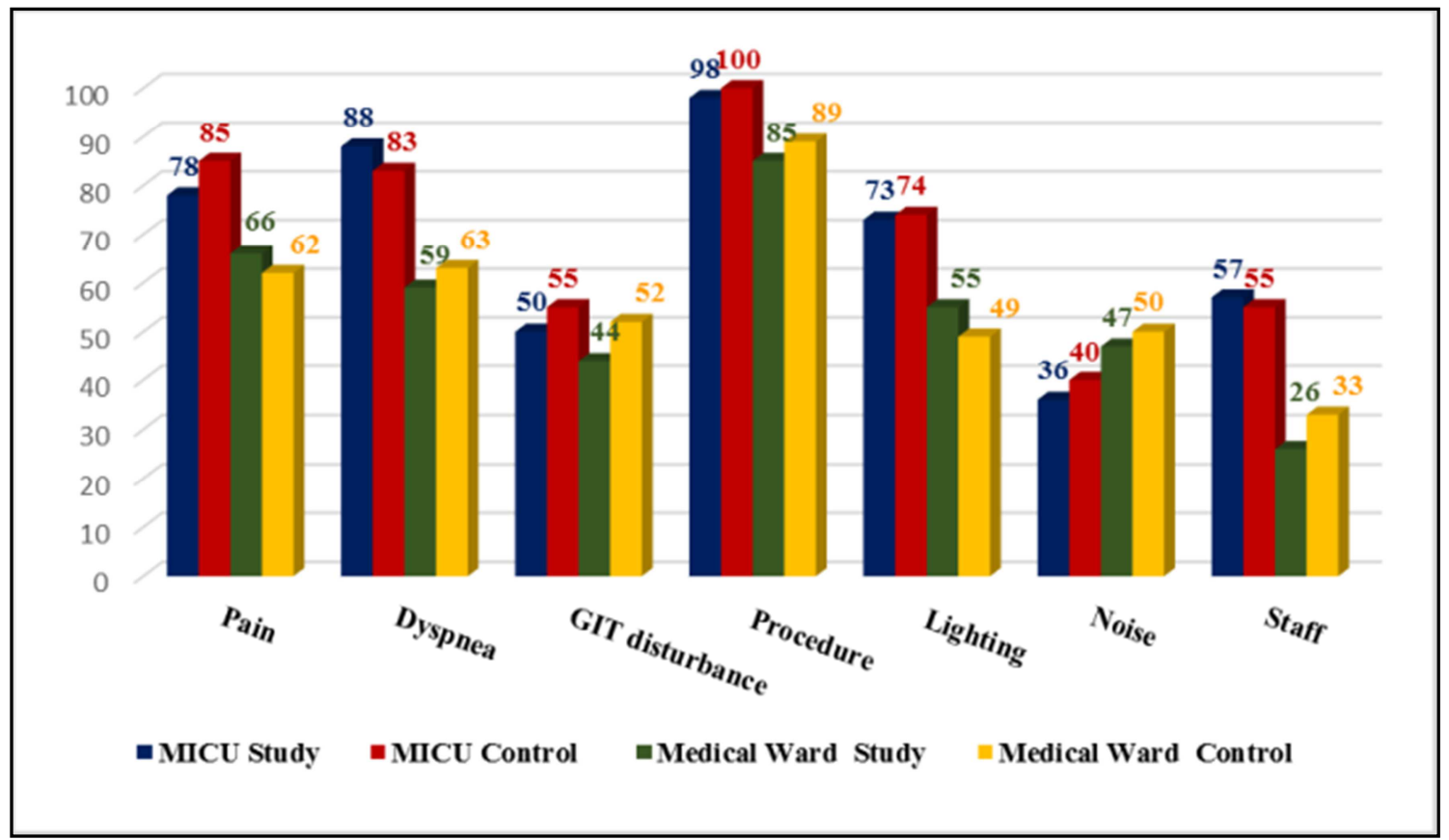

Figure 1. Percentage distribution of the factors that affect sleep quality among the studied groups. 
Table 2. Correlations between socio demographic data of the (Medical Ward and MICU patients) regarding Factors related to Patient in study and control groups.

\begin{tabular}{|c|c|c|c|c|c|c|c|c|}
\hline \multirow{4}{*}{ Socio Demographic data } & \multicolumn{8}{|c|}{ Factors related to Patient } \\
\hline & \multicolumn{4}{|c|}{ Medical Ward } & \multicolumn{4}{|l|}{ MICU } \\
\hline & \multicolumn{2}{|c|}{ Study $(\mathrm{N}=\mathbf{3 8})$} & \multicolumn{2}{|c|}{ Control $(\mathrm{N}=38)$} & \multicolumn{2}{|c|}{ Study $(\mathrm{N}=38)$} & \multicolumn{2}{|c|}{ Control $(\mathrm{N}=38)$} \\
\hline & $\mathbf{R}$ & $P$-value & $\mathbf{R}$ & $P$-value & $\mathbf{R}$ & $P$-value & $\mathbf{R}$ & $P$-value \\
\hline Age & .043 & .795 & -.148 & .375 & .178 & .284 & .055 & .743 \\
\hline Gender & .046 & .782 & $-.105-$ & .531 & .279 & .090 & .184 & .269 \\
\hline Medical diagnosis & .153 & .360 & $-.258-$ & .118 & $.509^{* *}$ & .001 & $-.244-$ & .140 \\
\hline Presence of Chronic disease & -.179 & .282 & .048 & .774 & .123 & .462 & $-.115-$ & .490 \\
\hline
\end{tabular}

Table 2. Shows that no significant correlation among patients in medical ward and in MICU in relation to patient factors that affected their sleep quality with the socio demographics data of them.

Table 3. Percentage distribution of both study \& control groups at the medical ward and MICU before using breathing exercise (N=152).

\begin{tabular}{|c|c|c|c|c|c|c|c|c|}
\hline \multirow{3}{*}{$\begin{array}{l}\text { Total score of sleep quality questionnaire before } \\
\text { applying breathing exercise (after the } 1^{\text {st }} \text { night). }\end{array}$} & \multicolumn{4}{|c|}{ Medical Word } & \multicolumn{4}{|c|}{$\overline{M I C U}$} \\
\hline & \multicolumn{2}{|c|}{ Study $(\mathrm{N}=38)$} & \multicolumn{2}{|c|}{ Control $(\mathrm{N}=38)$} & \multicolumn{2}{|c|}{ Study $(\mathrm{N}=38)$} & \multicolumn{2}{|c|}{ Control $(\mathrm{N}=38)$} \\
\hline & No. & $\%$ & No. & $\%$ & No. & $\%$ & No. & $\%$ \\
\hline Great sleep quality (28-36) & 5 & 13 & 6 & 15.7 & 2 & 5.2 & 4 & 10.5 \\
\hline Moderate sleep quality (19-27). & 7 & 18.4 & 5 & 13 & 1 & 2.6 & 7 & 18.4 \\
\hline Mild to moderate sleep problem (10-18). & 11 & 29 & 10 & 26 & 15 & 39.5 & 10 & 26 \\
\hline sleep problems seem to be severe. $(0-9 \mid)$ & 15 & 39 & 17 & 44.7 & 20 & 52.6 & 17 & 44.7 \\
\hline Test of significance & \multicolumn{2}{|c|}{$\chi^{2}=0.597$} & \multicolumn{2}{|c|}{$\mathrm{P}$ - value $0.89 \mathrm{~N} . \mathrm{S}$} & \multicolumn{2}{|c|}{$\chi^{2}=6.41$} & \multicolumn{2}{|c|}{$P$ value $=0.09 \mathrm{~N} . \mathrm{S}$} \\
\hline
\end{tabular}

Table 3. The table Found majority of study and control groups at the medical ward had sleep problems seem to be severe (0-9|) with percentage (39\% and 44.7\%) before applying breathing exercise. At the MICU also majority of study and control groups has a sever sleep problem also with percentage $(52.6 \%$ and $44.7 \%)$ respectively with no statistical significant $p$ value $(0.89 \& 0.09)$ respectively.

Table 4. Percentage distribution of both study \& control groups at the medical ward and MICU after using breathing exercise (N=152).

\begin{tabular}{|c|c|c|c|c|c|c|c|c|}
\hline \multirow{3}{*}{$\begin{array}{l}\text { Total score of sleep quality questionnaire after using } \\
\text { breathing exercise (after the } 5^{\text {th }} \text { night). }\end{array}$} & \multicolumn{4}{|c|}{ Medical Word } & \multicolumn{4}{|c|}{ MICU } \\
\hline & \multicolumn{2}{|c|}{ Study $(\mathrm{N}=38)$} & \multicolumn{2}{|c|}{ Control $(\mathrm{N}=38)$} & \multicolumn{2}{|c|}{ Study $(\mathrm{N}=38)$} & \multicolumn{2}{|c|}{ Control $(\mathrm{N}=38)$} \\
\hline & No. & $\%$ & No. & $\%$ & No. & $\%$ & No. & $\%$ \\
\hline Great sleep quality (28-36) & 17 & 44.7 & 4 & 10.5 & 19 & 50 & 4 & 10.5 \\
\hline Moderate sleep quality (19-27). & 9 & 23.4 & 5 & 13 & 11 & 29 & 5 & 13 \\
\hline Mild to moderate sleep problem (10-18). & 5 & 13 & 15 & 39.4 & 5 & 13 & 12 & 31.5 \\
\hline sleep problems seem to be severe. $(0-9 \mid)$ & 7 & 26.3 & 14 & 36.8 & 3 & 7.8 & 17 & 44.7 \\
\hline
\end{tabular}

Table 4. clarifies that $(44.7 \%)$ of the study group patients at the medical ward and (50\%) of the study group at MICU had great sleep quality after applying breathing exercise with score (28-36) and $(23.4 \%) \&(29 \%)$ of the same groups respectively had Moderate sleep quality with score (19-27).
High percentage of the control group in both ward and MICU had moderate to severe sleep problems. There was statistical significand difference between all groups presented with $\mathrm{P}$ value. $\left(0.001 *\right.$ and $\left.0.000^{*}\right)$ respectively

Table 5. Percentage distribution of the patients of both study \& control groups in medical ward and MICU as regarding to total score of NEECHAM CONFUSION SCALE after applying breathing exercise $(N=152)$.

\begin{tabular}{|c|c|c|c|c|c|c|c|c|}
\hline \multirow{3}{*}{$\begin{array}{l}\text { Total score of NEECHAM confusion scale after applying } \\
\text { the breathing exercise (after the } 5^{\text {th }} \text { night). }\end{array}$} & \multicolumn{4}{|c|}{ Medical Word } & \multicolumn{4}{|c|}{ MICU } \\
\hline & \multicolumn{2}{|c|}{ Study $(\mathrm{N}=38)$} & \multicolumn{2}{|c|}{ Control $(\mathrm{N}=38)$} & \multicolumn{2}{|c|}{ Study $(\mathrm{N}=38)$} & \multicolumn{2}{|c|}{ Control $(\mathrm{N}=38)$} \\
\hline & No. & $\%$ & No. & $\%$ & No. & $\%$ & No. & $\%$ \\
\hline 0-19 (Moderate to severe confusion or delirium) & 4 & 10.5 & 13 & 34.2 & 6 & 15.7 & 15 & 39.4 \\
\hline 20-24 (Mild or early development of confusion or delirium)) & 3 & 8 & 12 & 31.5 & 4 & 10.5 & 8 & 21 \\
\hline $25-26$ ("at high risk of confusion and delirium) & 8 & 21 & 4 & 10.5 & 8 & 21 & 9 & 23.6 \\
\hline a- 27-30 ("Not Confused," or normal function) & 23 & 60.5 & 9 & 23.6 & 20 & 52.6 & 6 & 15.7 \\
\hline Test of significance & \multicolumn{2}{|c|}{$\chi^{2}=17.6$} & \multicolumn{2}{|c|}{$P-$ value $=0.001 *$} & \multicolumn{2}{|c|}{$\chi^{2}=12.7$} & \multicolumn{2}{|c|}{$P-$ value $=0.005^{*}$} \\
\hline
\end{tabular}

Table 5 find that, the majority of the study groups patients in the medical ward and MICU had no Confused," or normal function with percentage $(60.5 \%$ and $52.6 \%)$ respectively after applying breathing exercise. On the other had more than half of the control groups at the same setting had mild and moderate to severe confusion or delirium. There was statistical significance difference between all groups presented by $\mathrm{P}$ value $(0.001 * \& 0.005 *)$. 


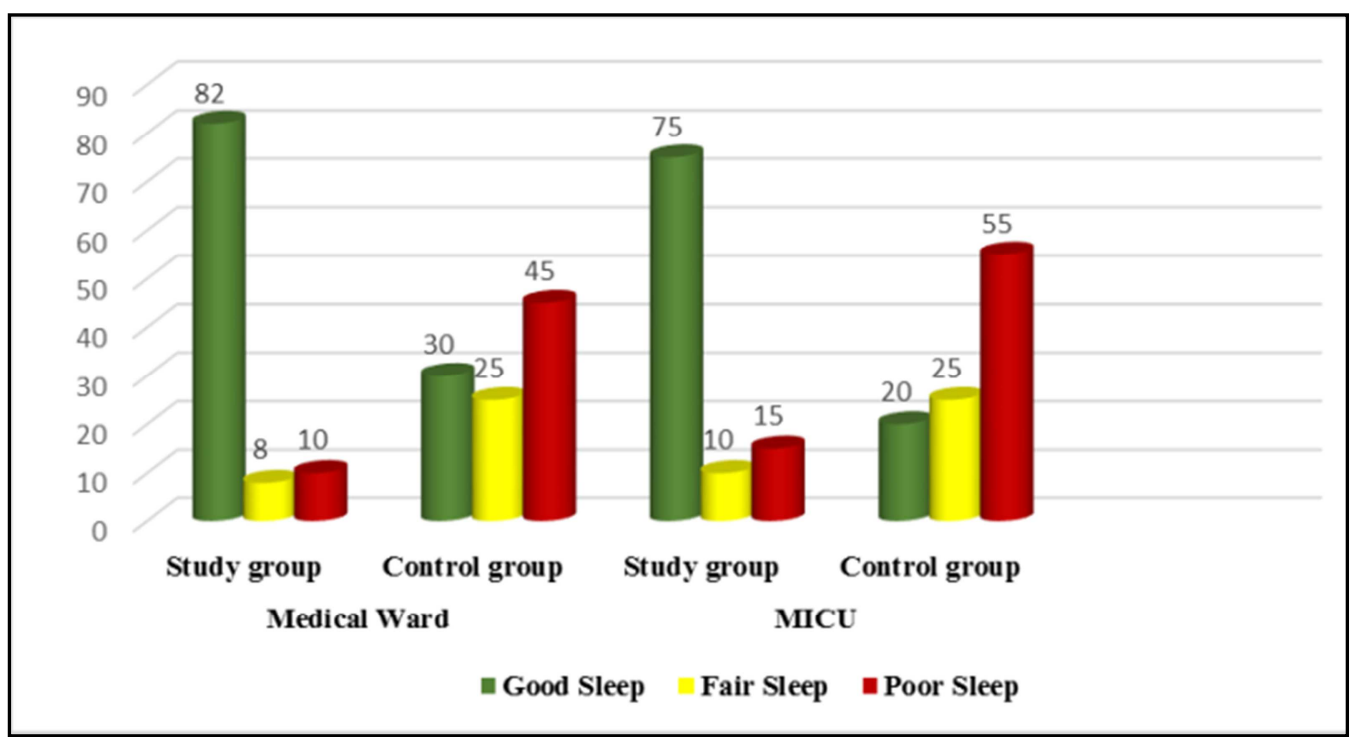

Figure 2. Percentage of patients in both study \& control groups regarding Patient's sleep pattern during hospitalization after applying breathing exercise ( $n=$ 152).

\section{Discussion}

Human being spend about one-third of their life in sleep: sleep is defined as a physiological state of reversible unconsciousness. Many references documented that more than $61 \%$ of hospitalized patients reported poor sleep quality. Causes of poor sleep quality are multifactorial. The main cause that prove poor sleep quality among hospitalized patients and especially at ICUs are primary illness and its pathophysiology play a significant role. The other causes that were high at the ICU environment were constant close monitoring, diagnostic testing, medical support such as mechanical ventilation or medication. Another environmental factors that present in both the general ward and ICUs were the excessive noise and light exposure which contribute to an un favorable sleep pattern [18].

Poor sleep quality cause various physical, cognitive and psychological complications. Nursing role for hospitalized patients should be directed to manage patient's needs, but sleeping quality did not have a standardized guideline to help nurses during their patient's care [19]. The present study was conducted to evaluate the effect of breathing exercise on sleep quality among hospitalized patients. The present study examined about 152 patients divided to 4 equal groups each group 38 patients. Two groups were sselected from the medical inpatient department (study and control) and the other two groups were selected from the medical intensive care unit (MICU).

The present study found the sociodemographic characteristics of the studied groups which revealed mean age and standard deviation (Mean $\pm \mathrm{SD}$ ) of the two medical ward groups (study and control) were (37.3 \pm 12.5 and $45.8 \pm$ 9.5 years) respectively and Mean \pm SD for age in the MICU groups (study and control) were (37.8 \pm 13.6 and $38.3 \pm 13.2$ years) respectively. Regarding to the gender more than half of the medical ward groups while the study group of the MICU were male, less than half of them were illiterate and small percentage of all the study groups were university educated patient. This result revealed that male at the middle age groups are at higher risk for hospital admission.

The present study in line with Alkan H. O., [20] they examined the influence of breathing exercise on sleep quality of heart failure patients with dyspnea, they found majority of the study groups were male and lower percentage of both study and control groups having higher educational level. The present study was also in line with Kulpatcharapong, [5] whom studied sleep quality among hospitalized patients, they found about half of the studied sample were male, they selected from the internal medical department (ordinary and private) and the medical ICU. This results prove that male were at higher risk for sleep disorder than female.

Patients of the present study had poor sleep quality because of variety of factors, as pain and dyspnea which were present in more than half of the medical ward and majority of the MICU groups. The result prove that pain and dyspnea are both stressful conditions that stimulate the sympathetic nervous system activity and leads to poor sleep quality. The present study was in line with Chauny, J. M., et al., [21]. whom reported that pain is one of the important patient's physiological factor that leads to poor sleep quality.

Invasive procedure cause sleep disturbance in a higher percentage among the MICU patient's groups (study and control) than the medical ward groups. The need for invasive procedure present in MICU because critical patients' needs frequent lab investigation collection and medication administration than the inpatient general medical ward which contribute to sleep disturbance. This study was in line with Altman, M. T., et al. [22] they found that hospitalized patients had sleep disturbance about (60) times at night because of the patient's care activities especially at ICUs.

The lighting as an environmental factor was present among 
the MICU than the medical ward groups, on the other hand the noise was present in a higher percentage at the medical ward than MICU. The lighting as an environmental factor present in the MICU because of machines as monitors, infusion, monitors and ventilators. This finding were in line with Hastings M. H., [23] they described that lighting decrease and suppress the release of melatonin, which is sleep-promoting factor which secreted from the pineal gland which controlled by the suprachiasmatic nuclei (SCN), the central circadian clock located in the hypothalamus.

Also Pisani, M. A., \& D’Ambrosio, C. [5] found high percentage of the hospitalized patient reported that light is the most common factor that leads to poor sleep quality they descriped lighting as patient's circadian rhythm disturbing factor. Also the present study results were in line with Ding, et al [8] reported that $(100 \%)$ of the MICU staff and all the participated patients experienced noise and unit lighting were the most sleepnig disturbance factors. Patients at the medical ward reported the staff (nurses and doctors) caused a lower percentage of sleep disturbance than that present in the MICU, this finding agree with Magdy M. D., [24] because they found that noise, lighting and then staffing factors were the most common activities that leads to poor sleep quality among patients admitted to the respiratory ICU.

Sleep assessment for hospitalized should be one of the standardized nursing activities to detect any presence of inadequate sleep quality and find the suitable nursing intervention based on it. The present study all groups were assessed for their sleep quality using sleep quality questionnaire once after first night and the second after the fifth night. Patient's assessment before applying the breathing exercise revealed that more than half of both medical ward groups (study and control) had moderate to severe sleep problems. On the other hand, MICU patients with poor sleep quality was present in the majority of cases before applying breathing exercise they had moderate to severe sleep problems. Which revealed that critical patients at higher risk for poor sleep quality than the general ward patients.

The present study was in line with Kulpatcharapong, [5] they found poor sleep quality present in a high prevalence among the internal medicine principal diagnoses including cardiopulmonary, infectious, gastrointestinal, hematologic, and other diseases were $57.7 \%, 35.7 \%, 55.7 \%, 71.4 \%$, and $33 \%$, respectively. Telias, I., \& Wilcox, E., [25] agree with the present study because critically ill patients experience circadian rhythm disturbance secondary to the absence of effective zeitgebers in the ICU environment. Telias, I., \& Wilcox, E [25] reported also that systemic inflammation may also disrupt circadian rhythmicity of chronobiologic markers. Also the noise and ICU environment get patients lack the REM stage of sleep.

Present study hypothesized that applying breathing exercise will improve sleep quality among hospitalized patients especially critical ill patients. Breathing exercise had positive effect on sleep quality which evaluated by using sleep quality questionnaire (SQQ). It results that majority of study group in the medical ward had great to moderate sleep quality than the control groups also the MICU study group about half of them had great sleep quality and about third of them had moderate sleep quality than the control group. the present study proves statistical significant difference between the study groups (medical ward and the MICU) and the control groups in the same settings. The present result proves the effect of breathing exercise on reducing sleep disturbance factors as pain, dyspnea by increasing the amount of tissue oxygenation which in turn reduce dyspnea and pain perception. Also breathing exercise is one of the relaxation technique methods, that reduce patients' perception to light and noise.

Lowe, H., et al. [26] prove the same result of the present study by considering breathing exercise and aerobics are the alternative non-pharmacological treatment of sleep pattern disturbance. Lowe, H., et al [26] prove that aerobic and breathing exercise improve patient's physical and mental health. The present study result agreed by Hasina, N. S., et al. [27] they prove that applying sleep hygiene and breathing exercise is an easy activity to apply for patients, breathing exercise reduces muscle tension, leads to bronchial dilatation and improves oxygenation to produce relaxation.

Hasina, N. S., et al. [27] prove that sleep quality and quality of life of the hemodialysis patients improved after applying breathing exercise with high significance of $(\mathrm{P}$ value 0.000). Yang, Y., S., et al., [28] examined the effect of exercise on sleep quality in pregnant woman, they reported that relaxation exercise as youga or breathing exercise and aerobic exercise is one of the non- pharmacological nursing intervention that will reduce insomnia and anxiety. On the other hand, Jerath, R., et al. [29] reported that breathing exercise result in stronger sympatho-inhibition that lead to reduction in pain intensity, dyspnea among asthmatic patient, improves cardiorespiratory synchronization and improves mood disorders which intern improves sleep quality.

Improving sleep quality will in turn reduce many pathological complications as delirium which is the second hypothesis in this study. The effect of good sleep quality on reducing delirium present in the following result, more than half of both medical ward and MICU study groups had no presence of delirium after applying the breathing exercise and their sleep quality were improved. Promoting good sleep improves central nervous system functions and breathing exercise enhances cerebral oxygen supply which leads to reduction in circadian rhythm disturbance and enhancing good sleep quality which improves cognitive functions and delirium reduction. The present study was supported by Lu, Y., et al. [30] they found that promoting sleep quality and keeping normal circadian health help prevent ICU delirium. Also Lu, Y., et al. [30] reported reduction in the risk of ICU delirium in study group those received sleep and circadian interventions by $50 \%$ compared with the risk among control patients.

Shalaby Khalaf Mahran., et al., [31] mentioned sleep disturbance and delirium, both of them has the same risk factors. Also Mahran, K. S. G., et al 2018 reported strong positive correlation between NEECHAM scale and sleep 
quality assessment which prove that any improvement in sleep quality among the study groups will cause reduction in delirium than the routine care groups.

Torres-Contreras, et al., [32] described that medication administration, environmental factor as ICU admission, invasive procedures and sleep deprivation were form the modifiable risk factors that leads to delirium. Lewandowska, K., et al., [33] reported that to effectively prevent and reduce delirium among hospitalized patients especially those at critical unit, nurses and the medical staff should implement effective guidelines to treat sleep disturbance and the modifiable risk factors that leads to it. Finally trying to assess sleep quality and implement measures to promote relaxation technique as breathing exercise leads to good sleep quality and delirium reduction which will have pitter effect on patient's outcome especially those at critical units.

\section{Conclusion}

Sleep quality level in hospitalized patients at the general wards and critical units is an important thing that nurses should consider. Trying to include breathing exercise in the routine nursing interventions would have been many positive effect as improving sleep quality, control many sleep disturbance factors and reducing the occurrence of delirium.

\section{Recommendations}

Patients should be assessed regularly during their hospitalization for their sleep quality.

Delirium prevention and early detection should be included in the daily nursing care.

Breathing exercise has positive effect for patients and should be included for every patient's education.

Nurses should be educated about the sleep assessment methods as sleep quality questionnaire

Nurses should learn delirium assessment technique and measures to prevent it.

Nurses should provide patients with health educations about breathing exercise and its benefits in reducing many complains.

Further research is needed on large sample about the use of relaxation technique methods and its effect on improving sleep quality and reducing the incidence of delirium.

\section{References}

[1] Rafay, H., Honarmand, K., Le, J. B., Mohan, S., Rochwerg, B., Skrobik, Y.,... \& Weinhouse, G. L. (2019). Systematic Review of Risk Factors Associated with Disrupted Sleep in Critically Ill Patients. In A69. SRN: WHAT CAN WE LEARN FROM CLINICAL AND EPIDEMIOLOGICAL STUDIES IN SLEEP? (pp. A2283-A2283). American Thoracic Society.

[2] Simons, K. S., Van den Boogaard, M., \& De Jager, C. P. (2019). Impact of intensive care unit light and noise exposure on critically ill patients. Neth J Crit Care, 27, 145-149.
[3] Romagnoli, S., Villa, G., Fontanarosa, L., Tofani, L., Pinelli, F., De Gaudio, A. R., \& Ricci, Z. (2019). Sleep duration and architecture in non-intubated Intensive Care Unit patients: An observational study. Sleep Medicine.

[4] Kulpatcharapong, S., Chewcharat, P., Ruxrungtham, K., Gonlachanvit, S., Patcharatrakul, T., Chaitusaney, B., \& Chirakalwasan, N. (2020). Sleep Quality of Hospitalized Patients, Contributing Factors, and Prevalence of Associated Disorders. Sleep Disorders, 2020.

[5] Pisani, M. A., \& D'Ambrosio, C. (2019). Sleep and Delirium in Critically Ill Adults: A Contemporary Review Chest. Chest.

[6] Mattiussi, E., Danielis, M., Venuti, L., Vidoni, M., \& Palese, A. (2019). Sleep deprivation determinants as perceived by intensive care unit patients: Findings from a systematic review, meta-summary and meta-synthesis. Intensive and Critical Care Nursing.

[7] Laghi, F., \& Shaikh, H. (2020). Clarifying the Effect of Sleep Deprivation on the Respiratory Muscles.

[8] Ding, Q., Redeker, N. S., Pisani, M. A., Yaggi, H. K., \& Knauert, M. P. (2017). Factors influencing patients' sleep in the intensive care unit: perceptions of patients and clinical staff. American Journal of Critical Care, 26 (4), 278-286.

[9] Pagnucci, N., Tolotti, A., Cadorin, L., Valcarenghi, D., \& Forfori, F. (2019). Promoting nighttime sleep in the intensive care unit: alternative strategies in nursing. Intensive and Critical Care Nursing, 51, 73-81.

[10] Heinrich, T. W., Kato, H., Emanuel, C., \& Denson, S. (2019). Improving the Validity of Nurse-Based Delirium Screening: A Head-to-Head Comparison of Nursing Delirium-Screening Scale and Short Confusion Assessment Method. Psychosomatics, 60 (2), 172-178.

[11] Tilouche, N., Hassen, M. F., Ali, H. B. S., Jaoued, O., Gharbi, R., \& El Atrous, S. S. (2018). Delirium in the intensive care unit: Incidence, risk factors, and impact on outcome. Indian journal of critical care medicine: peer-reviewed, official publication of Indian Society of Critical Care Medicine, 22 (3), 144.

[12] Ghorbani, A., Hajizadeh, F., Sheykhi, M. R., \& Asl, A. M. P. (2019). The Effects of Deep-Breathing Exercises on Postoperative Sleep Duration and Quality in Patients Undergoing Coronary Artery Bypass Graft (CABG): a Randomized Clinical Trial. Journal of Caring Sciences, 8 (4), 219 .

[13] Aksu, N. T., Erdogan, A., \& Ozgur, N. (2018). Effects of progressive muscle relaxation training on sleep and quality of life in patients with pulmonary resection. Sleep and Breathing, $22(3), 695-702$.

[14] Laver, K. E., Spargo, C., Saggese, A., Ong, V., Crotty, M., Lovato, N.,... \& Vakulin, A. (2020). Sleep Disturbance and Disorders within Adult Inpatient Rehabilitation Settings: A Systematic Review to Identify Both the Prevalence of Disorders and the Efficacy of Existing Interventions. Journal of the American Medical Directors Association.

[15] Wang, P., Song, L., Wang, K., Han, X., Cong, L., Wang, Y.,... \& Du, Y. (2020). Prevalence and associated factors of poor sleep quality among Chinese older adults living in a rural area: a population-based study. Aging Clinical and Experimental Research, 32 (1), 125-131. 
[16] Ibáñez, V., Silva, J., \& Cauli, O. (2018). A survey on sleep questionnaires and diaries. Sleep medicine, 42, 90-96.

[17] Neelon, V. J., Champagne, M. T., Carlson, J. R., \& Funk, S. G. (1996). The NEECHAM Confusion Scale: construction, validation, and clinical testing. Nursing research, 45 (6), 324330 .

[18] Schinkelshoek, M. S., van Luxemburg, R. P., Bes, A. L., Ottens, T. H., Fronczek, R., Lammers, G. J., \& van Westerloo, D. J. Good night and good luck: sleep in the ICU.

[19] Bevan, R., Grantham-Hill, S., Bowen, R., Clayton, E., Grice, H., Venditti, H. C.,... \& Hill, C. M. (2019). Sleep quality and noise: comparisons between hospital and home settings. Archives of disease in childhood, 104 (2), 147-151.

[20] Alkan, H. O., Uysal, H., Enç, N., \& Yigit, Z. (2017). Influence of breathing exercise education applied on patients with heart failure on dyspnoea and quality of sleep: a randomized controlled study. International Journal of Medical Research \& Health Sciences, 6 (9), 107-113.

[21] Chauny, J. M., Paquet, J., Carrier, J., Lavigne, G., Marquis, M., Cournoyer, A., \& Daoust, R. (2019). Subjective sleep quality and its etiology in the emergency department. Canadian Journal of Emergency Medicine, 21 (2), 249-252.

[22] Altman, M. T., Knauert, M. P., \& Pisani, M. A. (2017). Sleep disturbance after hospitalization and critical illness: a systematic review. Annals of the American Thoracic Society, 14 (9), 1457-1468.

[23] Hastings, M. H., Maywood, E. S., \& Brancaccio, M. (2018). Generation of circadian rhythms in the suprachiasmatic nucleus. Nature Reviews Neuroscience, 19 (8), 453-469.

[24] Magdy, D. M., Metwally, A., \& Makhlouf, H. A. (2019). Study of sleep quality among patients admitted to the respiratory intensive care unit. Egyptian Journal of Bronchology, 13 (1), 114.

[25] Telias, I., \& Wilcox, M. E. (2019). Sleep and circadian rhythm in critical illness. In Annual Update in Intensive Care and Emergency Medicine 2019 (pp. 651-664). Springer, Cham.
[26] Lowe, H., Haddock, G., Mulligan, L. D., Gregg, L., FuzellierHart, A., Carter, L. A., \& Kyle, S. D. (2019). Does exercise improve sleep for adults with insomnia? A systematic review with quality appraisal. Clinical psychology review, 68, 1-12.

[27] Hasina, S. N., Sukartini, T., \& Setiyowati, E. (2019). Effect Of Sleep Hygiene And Deep Breathing Exercise With Spiritual Care On Sleep Quality And Quality Of Life Of Hemodialysis Patient In Ahmad Yani Islamic Hospital Surabaya. Jurnal Ners dan Kebidanan Indonesia, 6 (2), 140-151.

[28] Yang, S. Y., Lan, S. J., Yen, Y. Y., Hsieh, Y. P., Kung, P. T., \& Lan, S. H. (2020). Effects of Exercise on Sleep Quality in Pregnant Women: A Systematic Review and Meta-analysis of Randomized Controlled Trials. Asian Nursing Research.

[29] Jerath, R., Beveridge, C., \& Barnes, V. A. (2019). Selfregulation of breathing as an adjunctive treatment of insomnia. Frontiers in psychiatry, 9, 780.

[30] Lu, Y., Li, Y. W., Wang, L., Lydic, R., Baghdoyan, H. A., Shi, X. Y., \& Zhang, H. (2019). Promoting sleep and circadian health may prevent postoperative delirium: a systematic review and meta-analysis of randomized clinical trials. Sleep medicine reviews.

[31] Shalaby Khalaf Mahran, G., Abd-Elshafy, S. K., El-hmeed, N. A. E., Abdel-Wahab Ibrahim, A. I., Gamal Eldeen AbdElHafez, N., \& Kadees Marzouk, H. The effect of night light on delirium occurrence in post-operative cardiac patients.

[32] Torres-Contreras, C. C., Páez-Esteban, A. N., del Castillo, A. H. D., Rincón-Romero, M. K., Amaris-Vega, A., \& MartínezPatiño, J. P. (2019). Factors associated with delirium in critical patients in a health institution in Bucaramanga, Colombia. Enfermería Intensiva (English ed.), 30 (1), 13-20.

[33] Lewandowska, K., Małkiewicz, M. A., Siemiński, M., Cubała, W. J., Winklewski, P. J., \& Mędrzycka-Dąbrowska, W. A. (2020). The role of melatonin and melatonin receptor agonist in the prevention of sleep disturbances and delirium in intensive care unit-a clinical review. Sleep Medicine. 